\title{
Patients with type 2 diabetes mellitus have an elevated prevalence of cardiovascular disease
}

Fernández-García, José Carlos (1), Cortés-Salazar, Carmen María (2)

1. Endocrinology Department. Virgen de la Victoria University Hospital. Málaga (Spain).

2. Badolatosa Primary Care Centre. Seville (Spain).

\section{Objectives}

To evaluate the prevalence of cardiovascular disease (CVD) and associated risk factors in patients with type 2 diabetes mellitus (T2DM) attended in a rural primary care setting.

\section{Basal characteristics $(\mathbf{n}-79)$}

\begin{tabular}{|l|c|}
\hline Age (years) & $70.1 \pm 11.8$ \\
Male (\%) & 63.3 \\
BMl $\left(\mathrm{kg} / \mathrm{m}^{2}\right)$ & $30.8 \pm 11.8$ \\
T2DM duration (years) & $5.9 \pm 5$ \\
Hba1c (\%) & $6.8 \pm 0.9$ \\
Triglycerides (mg/dl) & $148.3 \pm 81.1$ \\
Cholesterol (mg/dl) & $180.5 \pm 40.6$ \\
LDL-cholesterol $(\mathrm{mg} / \mathrm{dl})$ & $101.3 \pm 34.7$ \\
HDL-cholesterol $(\mathrm{mg} / \mathrm{dl})$ & $49.8 \pm 13.5$ \\
\hline
\end{tabular}

\section{Cardiovascular disease (\%)}

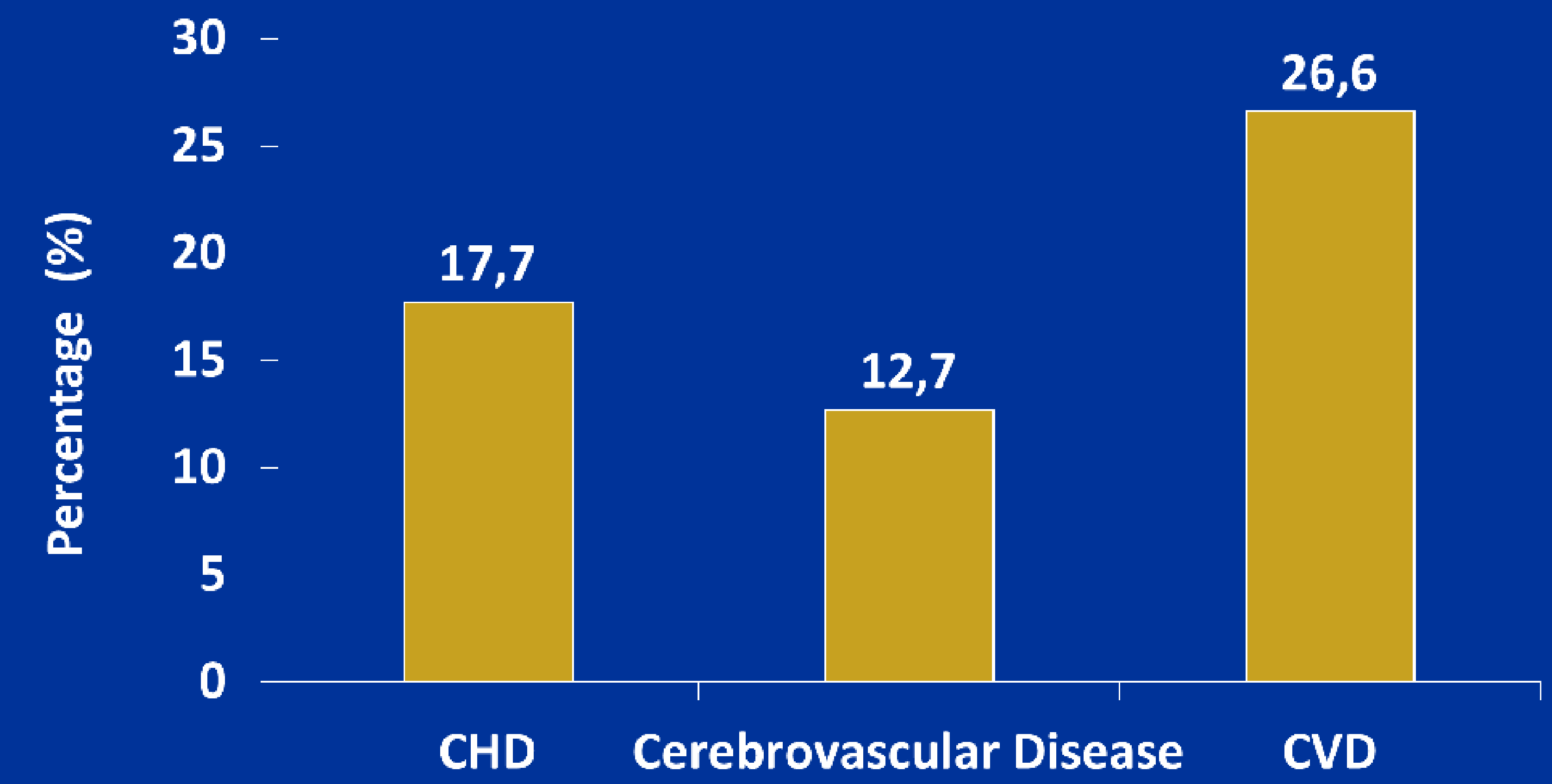

\section{Conclusions}

Most patients with T2DM attended in a rural setting have an elevated prevalence of cardiovascular risk factors and CVD is frequent, especially coronary heart disease.

\section{Methods}

- Cross-sectional study

- Inclusion criteria: T2DM patient followed in a primary care setting.

- Data about several cardiovascular risk factors was collected (age, sex, smoking, hypertension and dyslipidemia).

- Also, data about coronary heart disease (CHD) and cerebrovascular disease was gathered.

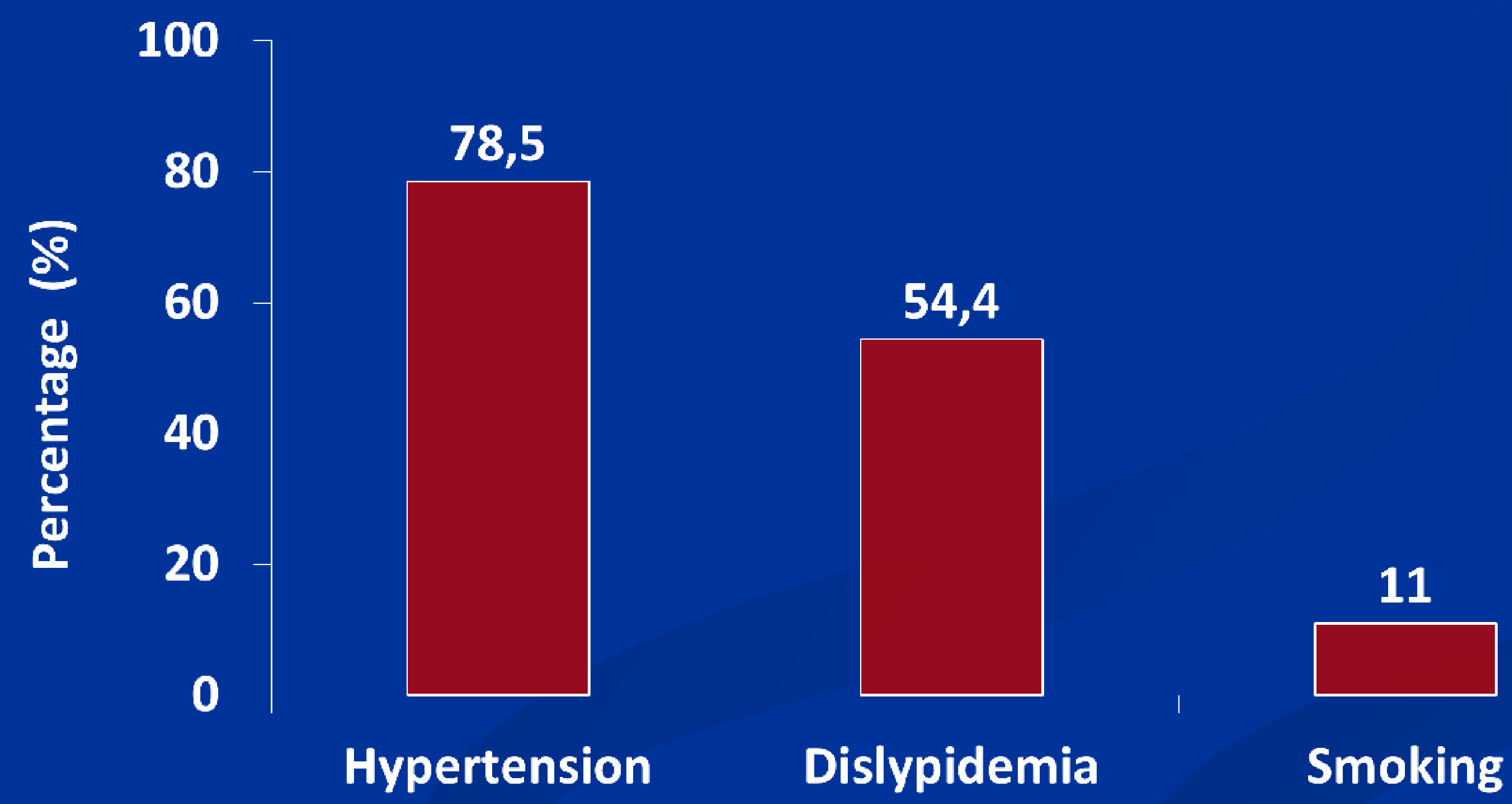

Patients characteristics by cardiovascular disease (\%)

\begin{tabular}{|l|c|c|c|}
\hline & CVD & CVD & $p$ \\
& $n-58$ & $n-21$ & \\
\hline Age (years) & $68.5 \pm 12.4$ & $74.2 \pm 8.9$ & 0.059 \\
Male/Female (\%) & $72 / 75.9$ & $28 / 24.1$ & $\mathrm{~ns}$ \\
BMI (kg/m²) & $30.9 \pm 6.2$ & $30.7 \pm 4$ & $\mathrm{~ns}$ \\
T2DM duration (years) & $5.5 \pm 4.9$ & $7.1 \pm 5.1$ & $\mathrm{~ns}$ \\
Hba1c (\%) & $6.7 \pm 0.9$ & $7.1 \pm 0.9$ & 0.11 \\
Triglycerides (mg/dl) & $143.8 \pm 69.2$ & $161.0 \pm 109.0$ & $\mathrm{~ns}$ \\
Cholesterol (mg/dl) & $184.1 \pm 37.9$ & $170.5 \pm 46.7$ & $\mathrm{~ns}$ \\
LDL-cholesterol (mg/dl) & $100.4 \pm 35$ & $102.6 \pm 34.7$ & $\mathrm{~ns}$ \\
HDL-cholesterol (mg/dl) & $51.5 \pm 13.2$ & $44.8 \pm 13.4$ & 0.064 \\
\hline
\end{tabular}

\section{Associated cardiovascular risk factors (\%)}

\begin{tabular}{|c|c|c|c|c|c|}
\hline 윳 472--EP & Diabetes (to include epidemiology, pathophysiology) & Poster & ECE2016 & 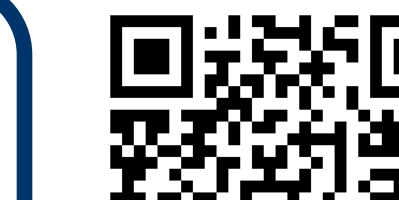 & Porter. \\
\hline & Jose Carlos & & 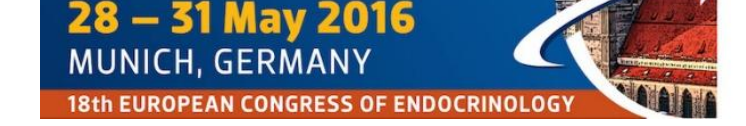 & 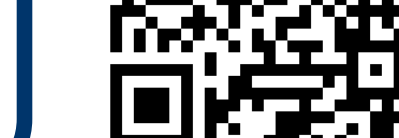 & Jessionunine \\
\hline
\end{tabular}

